\title{
Magnetic levitation performance of high-temperature superconductor over three magnetic hills of permanent magnet guideway with iron shims of different thicknesses
}

\author{
Yuming Gong • Gang Liang • Lifeng Zhao • \\ Yong Zhang $\cdot$ Yong Zhao $\cdot$ Xuyong Chen
}

Received: 3 December 2013/Revised: 27 March 2014/Accepted: 30 March 2014/Published online: 10 May 2014

(C) The Author(s) 2014. This article is published with open access at Springerlink.com

\begin{abstract}
Superconducting magnetic levitation performance, including levitation force and guidance force, is important for the application of high-temperature superconducting maglev. Both of them are not only affected by different arrays of superconductors and magnets, but also by the thickness of the iron shim between permanent magnets. In order to obtain the best levitation performance, the magnetic field distribution, levitation force, and guidance force of a new type of three magnetic hills of permanent magnet guideway with iron shim of different thicknesses $(4,6$, and $8 \mathrm{~mm})$ are discussed in this paper. Simulation analysis and experiment results show that the guideway with iron shim of $8 \mathrm{~mm}$ thickness possesses the strongest magnetic field and levitation performance when the suspension gap is larger than $10 \mathrm{~mm}$. However, with the decreasing of suspension gap, the guideway with iron shim of $4 \mathrm{~mm}$ thickness possesses the best levitation performance. The phenomena can be attributed to the density distribution of flux and magnetization of iron shim.
\end{abstract}

Keywords High-temperature superconductor - Three magnetic hills of permanent magnet guideway - Thickness of iron shim - Levitation force - Guidance force

Y. Gong · G. Liang · L. Zhao $(\varangle) \cdot$ Y. Zhang · Y. Zhao

Superconductivity and New Energy R\&D Center, Southwest

Jiaotong University, Chengdu 610031, China

e-mail: zhaolf@swjtu.edu.cn

X. Chen

Beijing Xinli Machinery Co, Ltd, Beijing 100039, China

\section{Introduction}

Since high-temperature superconducting (HTS) materials were found in 1986, people have paid much attention to the practical application of the permanent magnet-HTS levitation system. With the continuous development of superconducting technology [1-3], HTS maglev has been widely used in superconducting flywheel energy storage system [4], bearing [5, 6], HTS motors [7, 8], HTS cable [9], HTS maglev vehicle [10, 11], etc. On December 31, 2000, the world's first manned HTS maglev vehicle was successfully developed in Southwest Jiaotong University [11]. Subsequently, Russia and Germany developed their HTS maglev test vehicles in 2004 [12].

For HTS maglev vehicles, levitation and guidance force are very important parameters [13-19]. The improvement of levitation force can effectively enhance the carrying capacity of the maglev system. Guidance force directly determines the stability of the HTS maglev vehicles. Traditionally, permanent magnet guideway often possesses single magnetic hill [20], which cannot ensure the stability of HTS maglev vehicle running at high speed. In the meantime, researchers have paid much attention to the design of permanent magnet guideway, especially the permanent magnet arrangement in different ways [21-24]. However, little attention was paid to the iron shim inside guideway.

In this paper, the levitation and guidance force of hightemperature superconductor over the redesigned three magnetic hills of permanent magnet guideway with iron shim of different thicknesses are studied through simulation and experiments. The relationship between levitation performance and the thickness of iron shim obtained may provide a valuable reference for the optimum design of permanent magnet guideway for HTS maglev system. 


\section{Simulation analysis}

\subsection{Structure of permanent magnet guideway}

The guideway consists of four permanent magnets with section size of $40 \mathrm{~mm} \times 80 \mathrm{~mm}$, three iron shims, and two other irons used as clips to fix the whole structure, as shown in Fig. 1. The trademark of the permanent magnet is N45. The arrows in Fig. 1 indicate the direction of magnetization of magnets. Thus, three magnetic hills' distribution over the permanent magnet guideway can be obtained.

\subsection{Magnetic field simulation with Ansoft}

By simulating magnetic field with Ansoft Maxwell, we calculated the distribution of magnetic induction intensity $(B)$, as shown in Fig. 2. However, the actual magnetic induction intensity on the guideway surface is the same as that $2 \mathrm{~mm}$ above guideway in simulation [21], so the reference system was moved up $2 \mathrm{~mm}$.

\subsection{Magnetic field analysis}

The thicknesses of iron shim are set as 4,6 , and $8 \mathrm{~mm}$; and their guideways are marked with $a, b$, and $c$, respectively. The simulation results of $B_{Z}$ (vertical component of $B$ ) at different heights above the three guideways are shown in Fig. 3.

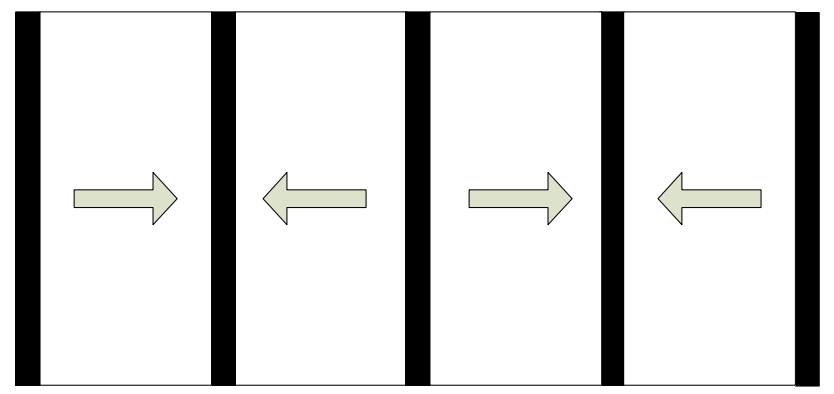

Fig. 1 The structure of permanent magnet guideway

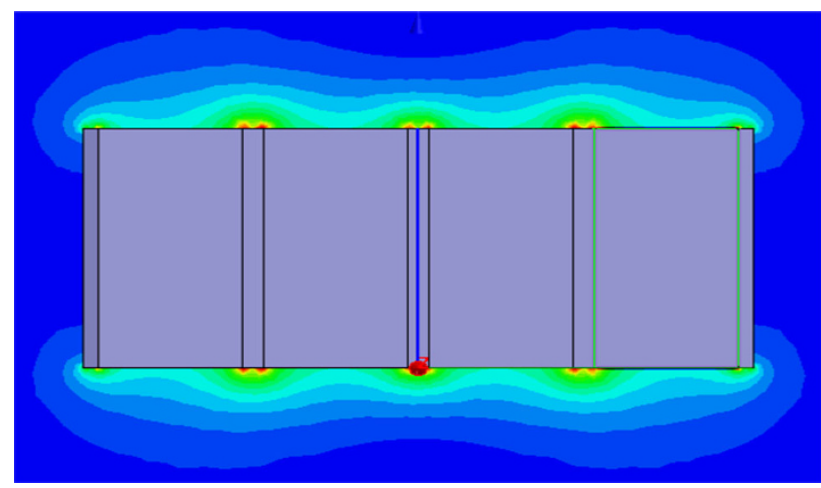

Fig. 2 Distribution diagram of magnetic induction intensity (a)

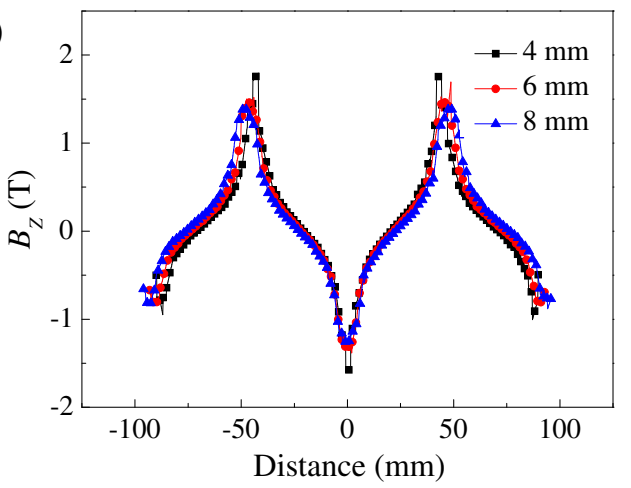

(b)

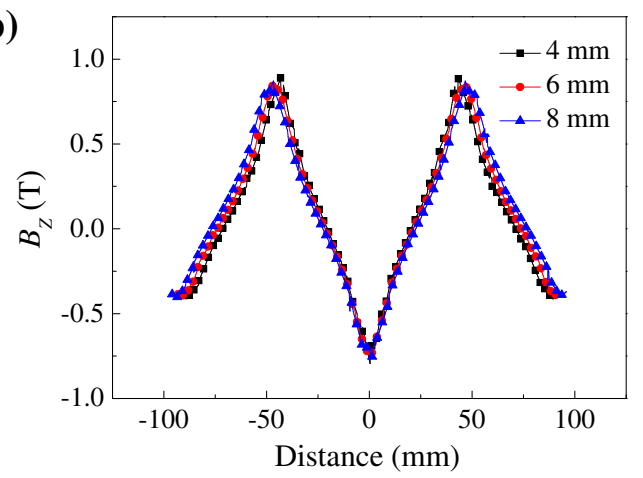

(c)

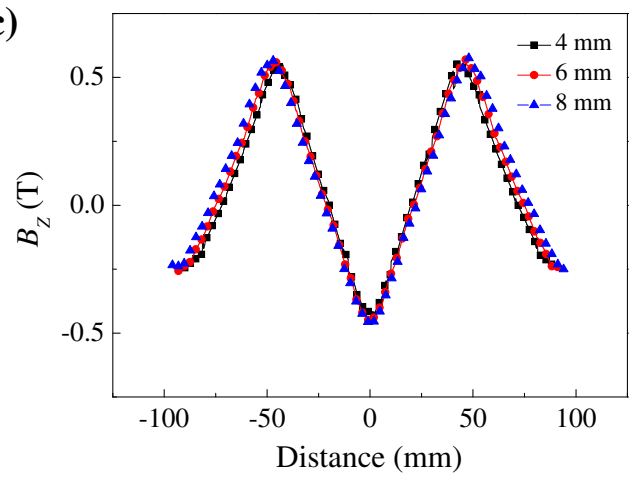

(d)

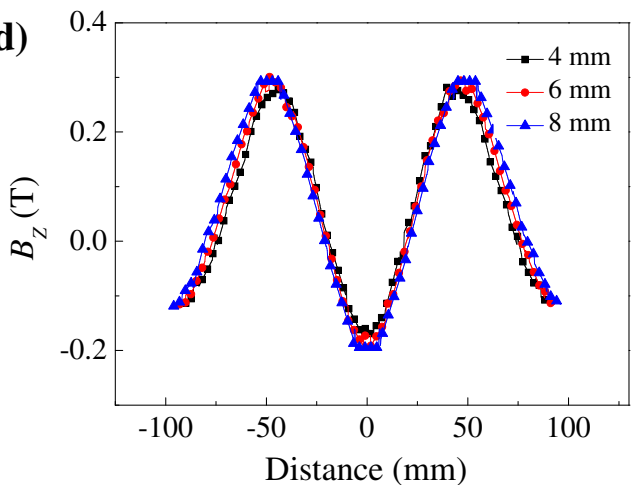

Fig. $3 B_{Z}$ distribution at different heights above guideways. a $0 \mathrm{~mm}$. b $5 \mathrm{~mm}$. c $10 \mathrm{~mm}$. d $20 \mathrm{~m}$

When the height is less than $5 \mathrm{~mm}$ above guideway, the $B_{Z}$ of the guideway $a$ with $4 \mathrm{~mm}$ iron shim is the largest, and it decreases with the height increasing. When the 
height is larger than $5 \mathrm{~mm}$, with the increase of height, $B_{Z}$ above guideway $a$ is the least, while that above guideway $c$ turns to be the largest. As we know, larger density of flux causes larger $B_{Z}$, and this should be the reason that the $B_{Z}$ of the iron shim in $4 \mathrm{~mm}$ thickness is the largest when the height is less than $5 \mathrm{~mm}$. However, the iron shim is magnetized by permanent magnets and presents flux. Thus the thicker iron shim should provide more flux. Furthermore, according to the magnetization curve of the iron shim, a thinner iron shim produces less flux than a thicker one in its saturation magnetization zone. This may cause $B_{Z}$ above guideway $c$ to be the largest when the height is larger than $5 \mathrm{~mm}$.

Figures 4 and 5 show the distribution of $B_{Z}$ above the middle and side iron shims, respectively, which indicate similar characteristics of $B_{Z}$ distribution for iron shims in different place. Nevertheless, it also can be noticed that the $B_{Z}$ of side iron shims is a little larger than that of the middle one.

As the levitation force is associated to the $B_{Z}$, we use $B_{Z}$ to estimate the levitation force of the HTS bulks and magnet levitation system. We take the levitation force $F_{\mathrm{L}}$ simply as proportional to $B_{Z}$ [14], namely $F_{\mathrm{L}} \propto B_{Z}$.

\section{Experiment}

\subsection{Tests of levitation force}

Three YBCO superconducting bulks were placed above the three magnetic hills, as shown in Fig. 6. Superconducting bulks were located in a container and cooled with liquid nitrogen.

One of the real guideways applied in experiments is shown in Fig. 7.

The HTS bulks placed inside container were cooled at different heights $(15,20,25$, and $30 \mathrm{~mm})$ and pressed to $5 \mathrm{~mm}$ above guideway, and then returned to the original locations, respectively. The container was moved down and up at a speed of $50 \mathrm{~mm} / \mathrm{min}$. The variations of the

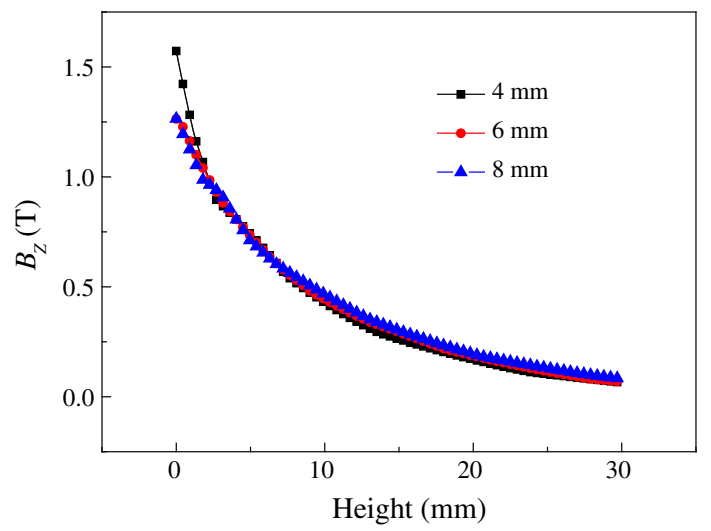

Fig. $4 B_{Z}$ distribution above middle iron shim average levitation force with distance for HTS bulks are shown in Fig. 8.

According to the experimental results, no matter what the cooling height is, the levitation force of guideway $a$ is less than that of the others. Analyzing the experimental data in detail, we found that the levitation force of

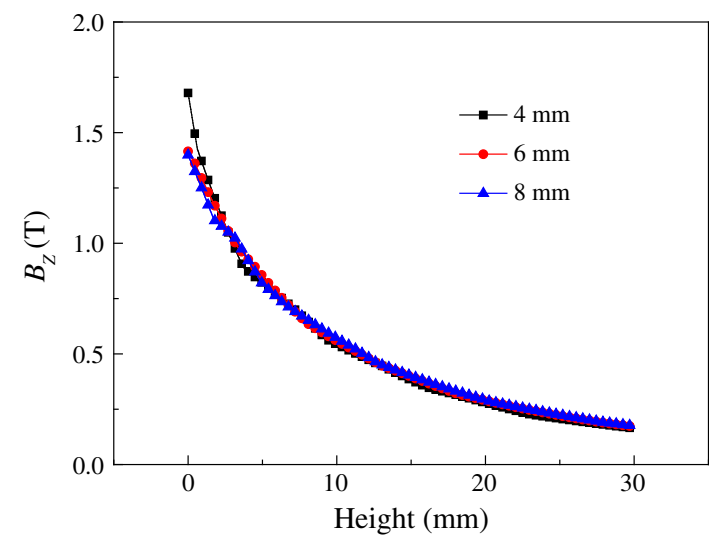

Fig. $5 B_{Z}$ distribution above side iron shim

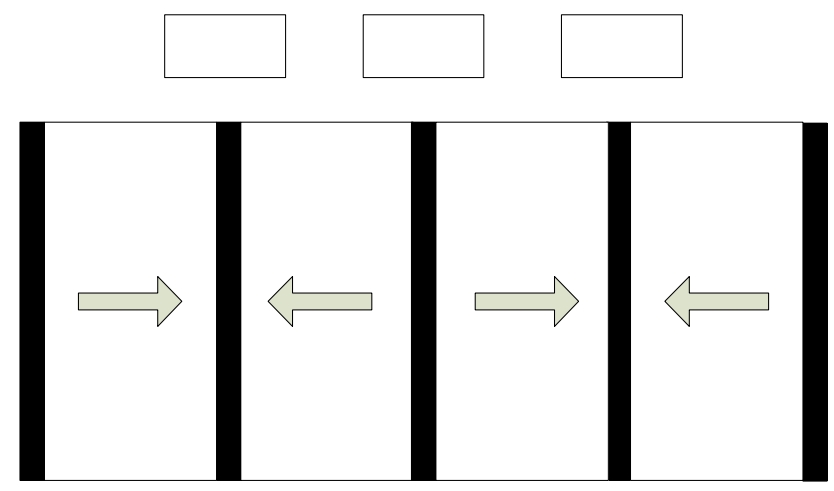

Fig. 6 HTS bulks above guideway

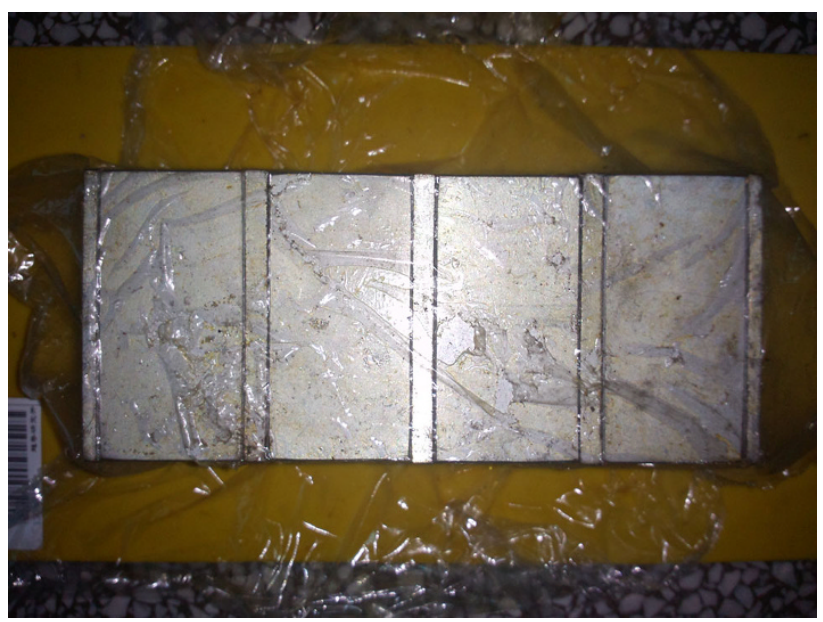

Fig. 7 Photography of experiment guideway $c$ 
(a)

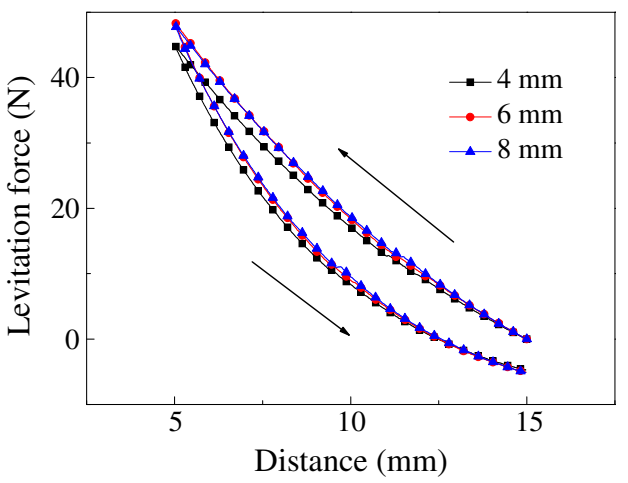

(b)

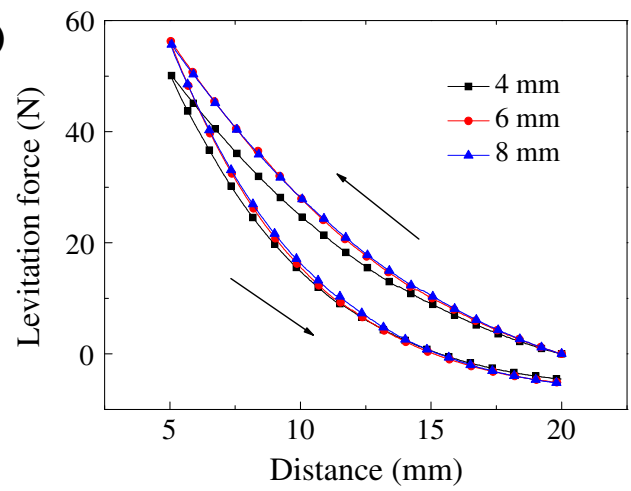

(c)

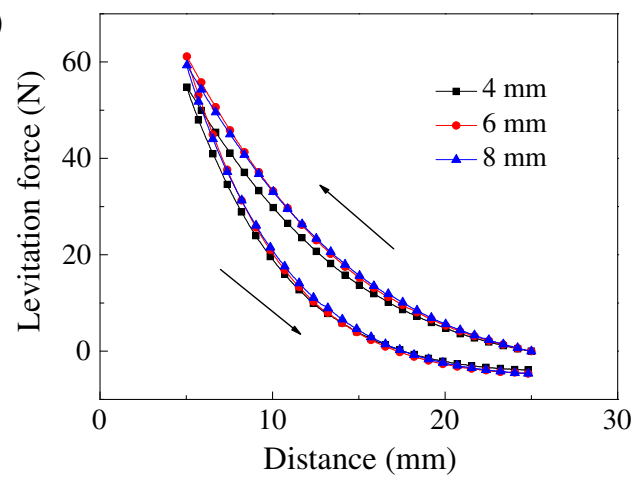

(d)

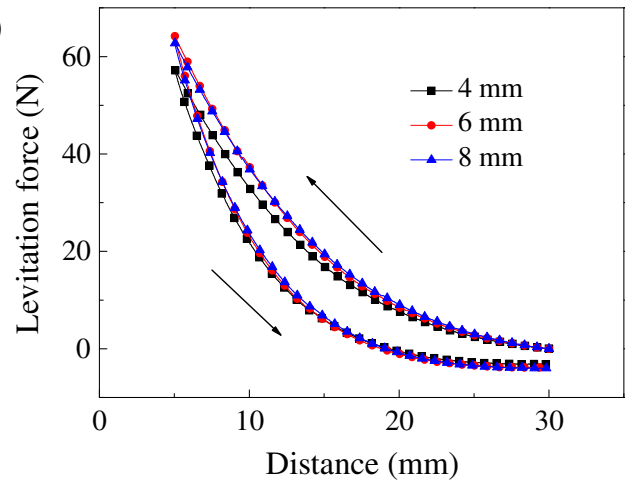

Fig. 8 Levitation force over different guideways with different cooling heights. a $15 \mathrm{~mm}$. b $20 \mathrm{~mm}$. c $25 \mathrm{~mm}$. d $30 \mathrm{~mm}$
Table 1 The actual $B_{Z}$ distribution above middle iron shim

\begin{tabular}{lccc}
\hline Height $(\mathrm{mm})$ & \multicolumn{3}{l}{$B_{Z}(\mathrm{G})$} \\
\cline { 2 - 4 } & Guideway $a$ & Guideway $b$ & Guideway $c$ \\
\hline 0 & 14,022 & 12,911 & 10,746 \\
5 & 7,366 & 7,453 & 7,357 \\
10 & 4,445 & 4,564 & 4,630 \\
15 & 2,703 & 2,905 & 3,136 \\
20 & 1,811 & 1,985 & 2,088 \\
25 & 1,064 & 1,268 & 1,373 \\
30 & 707 & 803 & 936 \\
\hline
\end{tabular}

Table 2 The actual $B_{Z}$ distribution above side iron shims

\begin{tabular}{lccc}
\hline Height $(\mathrm{mm})$ & \multicolumn{3}{l}{$B_{Z}(\mathrm{G})$} \\
\cline { 2 - 4 } & Guideway $a$ & Guideway $b$ & Guideway $c$ \\
\hline 0 & 14,815 & 13,252 & 11,476 \\
5 & 8,024 & 8,286 & 8,023 \\
10 & 4,916 & 5,081 & 5,159 \\
15 & 3,324 & 3,484 & 3,619 \\
20 & 2,303 & 2,474 & 2,568 \\
25 & 1,775 & 1,867 & 1,981 \\
30 & 1,297 & 1,362 & 1,469 \\
\hline
\end{tabular}

guideway $b$ is a little larger than that of guideway $c$ when the distance is less than $7 \mathrm{~mm}$. With the increase of distance, however, the levitation force of guideway $b$ become less than that of guideway $c$.

We further measured the vertical distribution of the $B_{Z}$ in the center of middle iron shim and side iron shim from surface to the height of $30 \mathrm{~mm}$. The results are shown in Tables 1 and 2.

$B_{Z}$ distributions and their corresponding levitation force imply that higher $B_{Z}$ causes larger levitation force. Comparing the distribution with levitation force, we take the relationship between them as $F_{\mathrm{L}} \propto B_{Z}$ on the whole. It is obvious that the extra $B_{Z}$ contributed by magnetized iron shim plays a key role in the process.

\subsection{Tests of guidance force}

HTS bulk over the permanent magnet guideway possesses guidance force when it is cooled with liquid nitrogen and captures a certain number of fluxes [16]. Guidance force has much to do with the field cooling height $[16,17]$, so the test height was set as the field cooling height of 15, 20, and $25 \mathrm{~mm}$, respectively. The field cooling superconductor bulks were pushed along the horizontal direction by the 


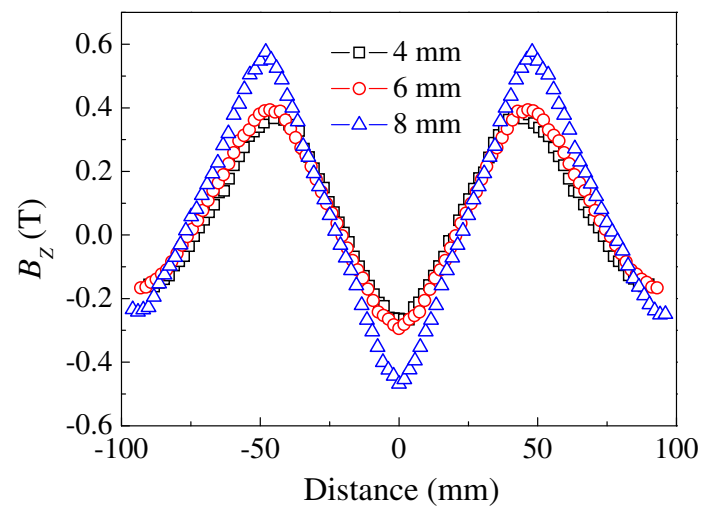

(a)

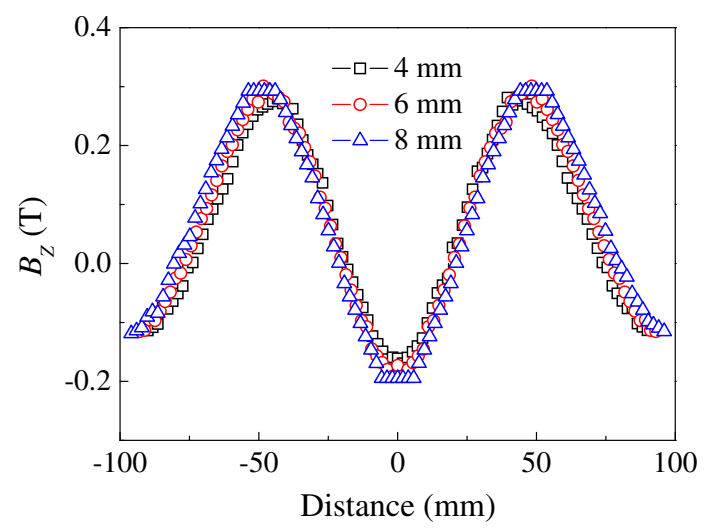

(b)

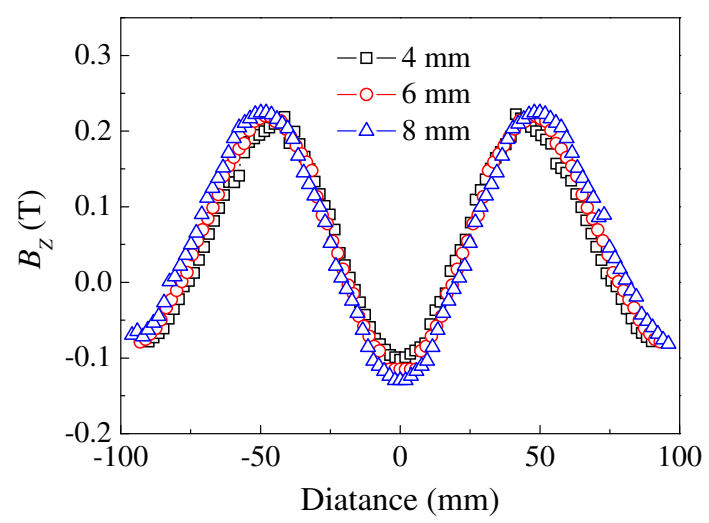

(c)

Fig. 9 Distribution of $B_{Z}$ above guideways with different field cooling heights. a $15 \mathrm{~mm}$. b $20 \mathrm{~mm}$. c $25 \mathrm{~mm}$

stepper motor connected with a force sensor. The guideways $a, b$, and $c$ were also used for the tests.

As we know, the guidance force is related to the magnetic flux captured by high-temperature superconductor, when it achieves the superconducting state [16, 17]. It can be seen from Fig. 9 that the $B_{Z}$ of $c$ is the largest among the three guideways. The higher the density of magnetic flux,

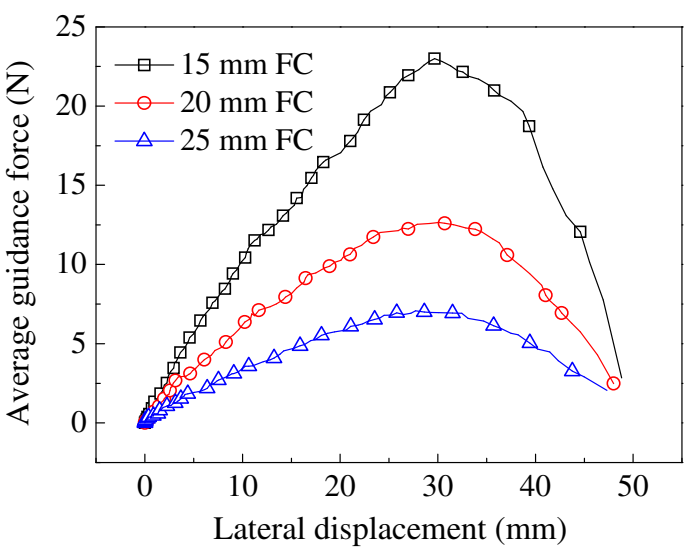

(a)

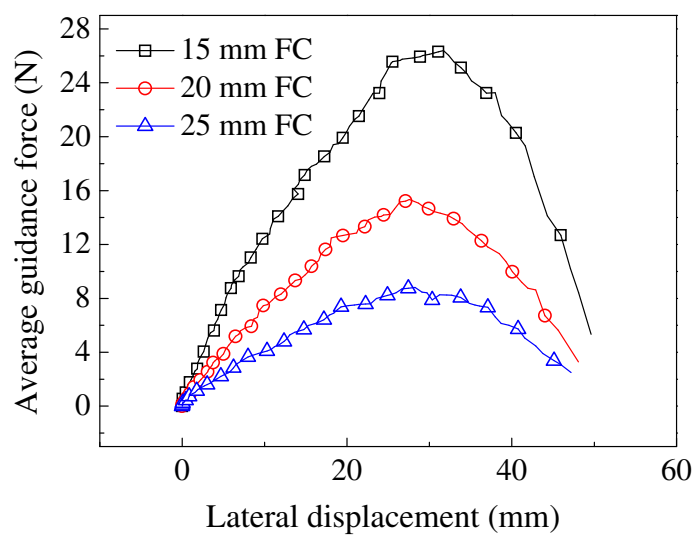

(b)

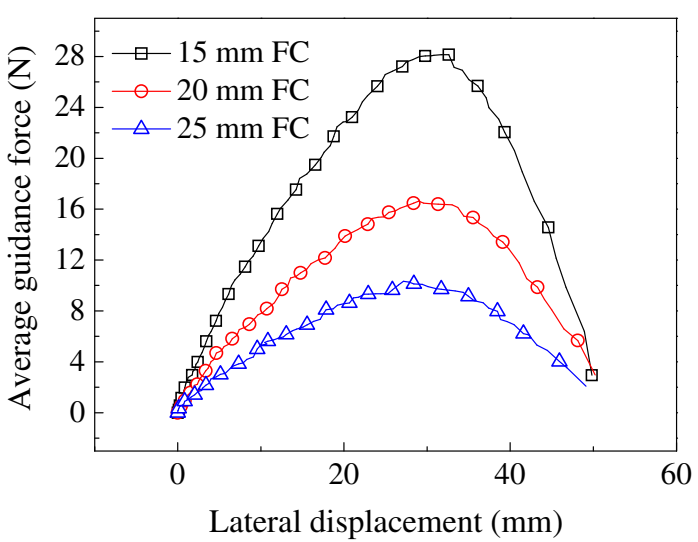

(c)

Fig. 10 Average guidance force of superconductor with different field cooling heights over the same guideway. a guideway $a$. b guideway $b$. c guideway $c$

the bigger the $B_{Z}$, and hence the larger guidance force. This rule is also reflected in Fig. 10.

Figure 10 shows the average guidance force of superconductor with different field cooling heights over the same guideway. The position where the superconductor 


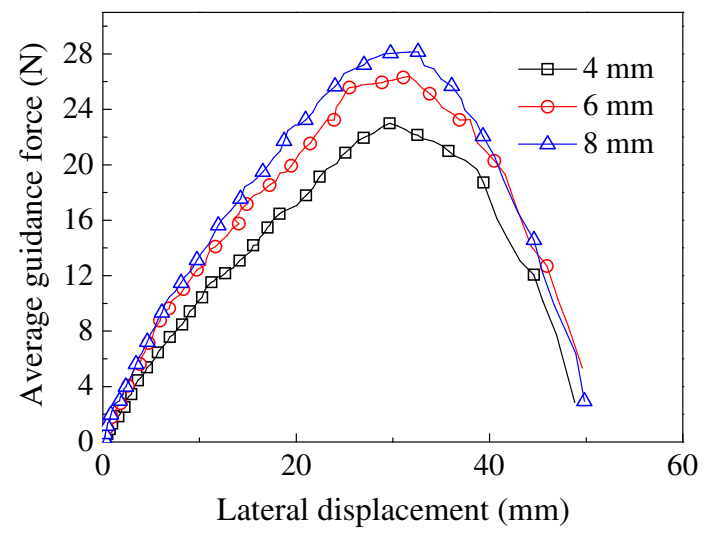

(a)

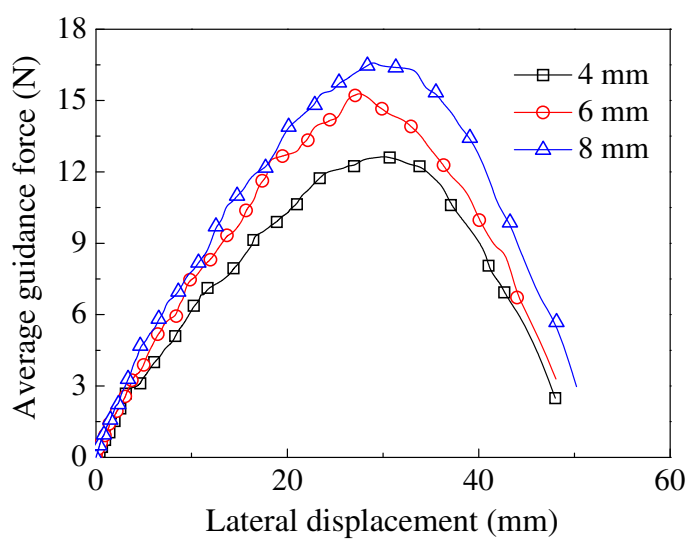

(b)

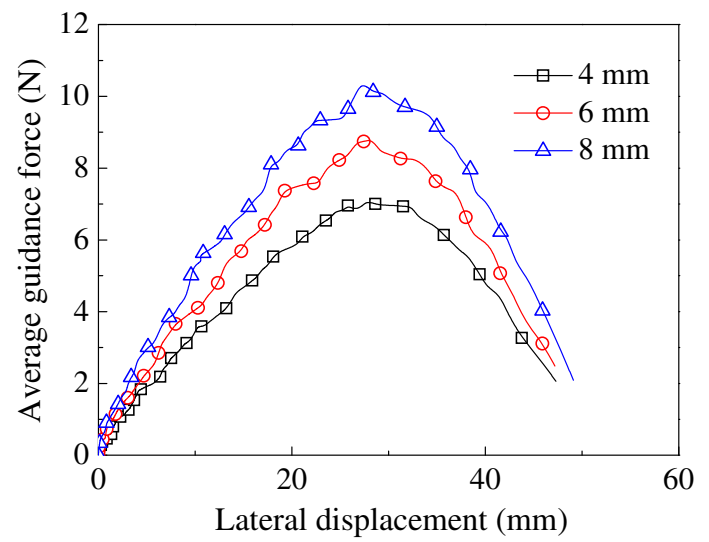

(c)

Fig. 11 Average guidance force of superconductor with different field cooling heights over different guideways. a $15 \mathrm{~mm}$. b $20 \mathrm{~mm}$. c $25 \mathrm{~mm}$

was cooled is regarded as zero in displacement. We can see that when the displacement is less than $20 \mathrm{~mm}$, namely half the size of a single permanent magnet, the guidance force is almost linear with the lateral displacement. When it is larger than $20 \mathrm{~mm}$, with the displacement increasing, the guidance force gradually tends to saturation and decline at last. Otherwise, with the decrease of field cooling height, the performance of guidance force gets better, which is similar to that described in literature $[16,17]$ for the single magnetic hill guideway and Halbach twin hills permanent magnet array.

The average guidance forces of superconductor bulks for different field cooling heights over different guideways are shown Fig. 11. We can see that, for the same field cooling height of 15 and $20 \mathrm{~mm}$, the thicker iron shim causes a larger guidance force. Moreover, the decrease of cooling height enlarges guidance force obviously. On the other hand, the decrease of the field cooling height will reduce the performance of HTS levitation force [16].

The main reason for the phenomenon observed above is due to the increase of captured flux with the iron shim getting thicker or the decrease of cooling height. The macro equivalent current density $J$, which is composed of eddy currents to maintain flux quantum beam in the numerous flux pinning centers, was enhanced with increase of the initial captured flux. The guidance force of HTS bulk will thus increase according to the guidance force calculation formula [16]:

$F_{\mathrm{G}}=\int_{V} J_{Y} B_{Z} \mathrm{~d} V$,

where $F_{\mathrm{G}}$ is guidance force, $J_{Y}$ stands for the horizontal component of $J$, and $V$ is the volume of the HTS bulk.

\section{Conclusion}

In this paper, simulation of the vertical component of magnetic induction intensity $\left(B_{Z}\right)$ is combined with experiments on levitation performance to discuss the variation of guidance force and levitation force with the thickness of iron shim and cooling height. Narrower iron shim may cause stronger $B_{Z}$ on the surface of guideway, but does not in the space. On the contrary,wider iron shim provides stronger $B_{Z}$ at higher height above guideway. Actually, the working height of superconducting bulks is usually not on the surface of guideway. Thus, it is very important to choose a proper thickness of iron shim to obtain the strongest $B_{Z}$ and the best levitation and guidance performance according to the different working height. This is meaningful for the application of HTS magnet levitation system.

Acknowledgments This work was supported by the National Magnetic Confinement Fusion Science Program (2011GB112001), the Program of International S\&T Cooperation (S2013ZR0595), the National Natural Science Foundation of China (No. 51271155), the Fundamental Research Funds for the Central Universities (SWJTU11CX073, SWJTU11ZT16 and SWJTU11ZT31), and the Science Foundation of Sichuan Province (2011JY0031 and 2011JY0130). 
Open Access This article is distributed under the terms of the Creative Commons Attribution License which permits any use, distribution, and reproduction in any medium, provided the original author(s) and the source are credited.

\section{References}

1. Yang TX (2008) Status of research in high temperature superconductivity technology in china. J China Acad Electron Inf Technol 3(2):122-127

2. Jiang ZJ (2009) Development of high temperature superconductor applications. Cryog Supercond 37(10):17-21

3. Jin JX (2008) High temperature superconductivity in the past twenty years part 2 -towards to practical applications. J Electron Sci Technol China 6(2):237-254

4. Suzuki T, Suzuki H, YasakaY, et al (1993) Adv Supercond (6): 1937

5. Fukuyama H, EndoS, TakizawaT, et al (1993) Adv Supercond (6): 1341

6. Deng ZG (2009) Research and development status of high temperature superconducting magnetic bearings. Trans China Electrotech Soc 24(9):1-8

7. Zheng LH (2007) Developments of high temperature superconducting motors. Electric Mach Control Appl 34(3):1-6

8. Xu YY (2013) A survey of the development of the low and high Tc superconducting motors/generators. Chin J Low Temp Phys 35(1):43-51

9. He Q (2013) A summarization of R \& D on HTS cable. Cryogen Superconduct 41(5):31-35

10. Wang JS, Wang SY (2005) Synthesis of bulk superconductors and their properties on permanent guideway. Frontiers Superconduct Mater 4:885-912

11. Wang JS, Wang SY (2002) The first man-loading high temperature superconducting maglev test vehicle in the world. Physica 378-381:809-814
12. Schultz L, De Haax O, Verges P et al (2005) Superconductively levitated transport system - the SupraTrans project. Appl Supercond 15(2):2301-2305

13. Deng ZG (2008) Two kinds of potential application of superconducting bulk magnet for high temperature superconducting maglev vehicle. Rare Metal Mater Eng 37(A04):350-354

14. Ren ZY (2002) Studying about relation between levitation force of $\mathrm{YBaCuO}$ Bulk over NdFeB guideway and magnetic field and magnetic field gradient. Chin J Low Temp Phys 24(4):293-297

15. Ren ZY (2000) Levitation force of multi-block YBaCuO high temperature superconductors over a permanent magnetic railway. Cryogen Superconduct 28(2):17-21

16. Ren ZY (2004) Experimental investigation and numerical calculation on high temperature superconducting maglev based on a permanent magnetic guideway. Dissertation, southwest Jiaotong University

17. Qin YJ (2009) Study on the guidance force characteristics of bulk high- $T_{\mathrm{c}}$ superconductor over permanent magnetic guideway by experiment and simulation. Dissertation, southwest Jiaotong University

18. Wang XR (2003) Research on guidance force of high temperature superconductor YBCO bulks over the permanent magnet guideway. Dissertation, southwest Jiaotong University

19. Qin GP (2010) Study of the relationship between levitation force and the arrangement of high temperature superconductivity bulks over guideway. Chin J Low Temp Phys 32(4):257-261

20. Halbach K (1980) Nucl Instrum Methods 169:1-10

21. Liang X (2012) Simulation and optimization of permanent magnet guideway arrangement based on ansoft. Cryogen Superconduct 41(3):49-52

22. Guo F (2008) Structural parameter optimization design for HTS permanent maglev orbit. Rare Metal Mater Engineer 37(A04):385-388

23. Wang LL (2007) The levitation performance of YBCO above three permanent magnet guideways. Cryogen Superconduct 35(4): 300-303

24. Dang QH (2011) PMG optimization design based on HTS bulk magnetic stiffness characteristic. Cryogen Superconduct 39(7):32-35 